\title{
Composición de comunidades y filoespecificidad de ectoparásitos de murciélagos en paisajes agropecuarios de Veracruz, México.
}

\section{Community composition and phylospecificity of bat ectoparasites in agricultural landscapes in Veracruz, Mexico.}

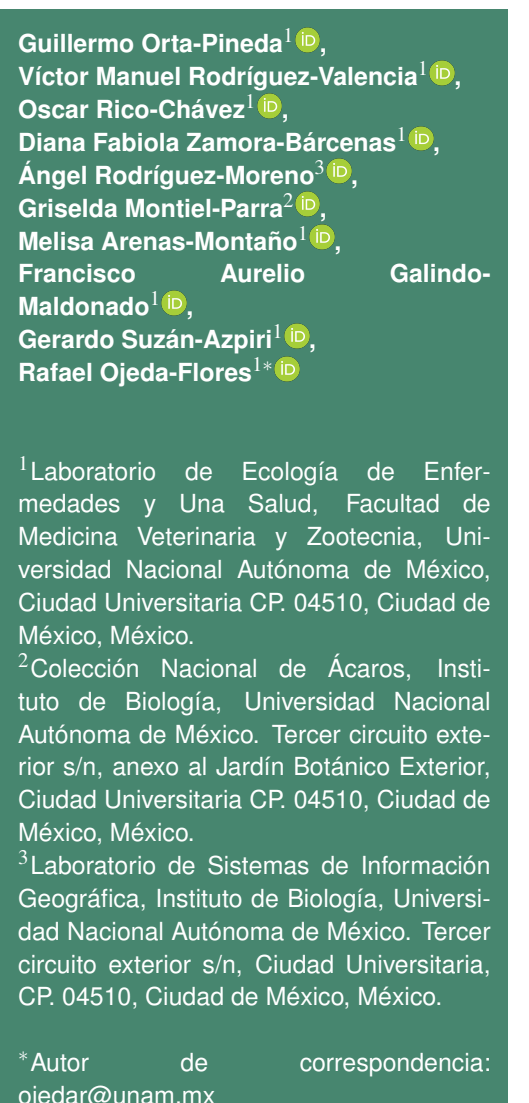

ojedar@unam.mx

Artículo científico

Recibido: 27 de noviembre de 2018

Aceptado: 07 de octubre de 2019

Como citar: Orta-Pineda G, RodríguezValencia VM, Rico-Chávez O, ZamoraBárcenas DF, Rodríguez-Moreno A, Montiel-Parra G, Arenas-Montaño M, Galindo-Maldonado FA, Suzán-Azpiri G, Ojeda-Flores R (2020) Composición de comunidades y filoespecificidad de ectoparásitos de murciélagos en paisajes agropecuarios de Veracruz, México. Ecosistemas y Recursos Agropecuarios 7(1): e2059. DOI: 10.19136/era.a7n1.2059
RESUMEN. Se evaluó la influencia de la transformación antropogénica del paisaje por actividades pecuarias sobre la composición y especificidad de las comunidades de ectoparásitos asociadas a murciélagos, en ranchos ganaderos del estado de Veracruz. Se compararon sitios con diferente nivel de transformación del paisaje que incluyeron producciones bajo sistemas de pastoreo en acahual, silvopastoril y pastizal. Se capturaron 944 murciélagos de 23 especies en ocho unidades de producción pecuaria. Se realizó la identificación taxonómica de 1433 ectoparásitos de 35 especies obtenidos a partir de 428 hospederos. Se realizaron análisis de diversidad taxonómica y especificidad filogenética entre las asociaciones murciélago-ectoparásito. La composición de las comunidades de ectoparásitos asociadas con Artibeus jamaicensis mostró diferencias significativas entre los tres niveles de transformación del paisaje. El acahual, que es el paisaje con menor transformación antropogénica, presentó los valores más altos de diversidad de especies de murciélagos y de ectoparásitos. La filoespecificidad de los estréblidos no presentó cambios significativos entre los diferentes tipos de paisaje del estudio. La consideración de factores antropogénicos que afecten la riqueza, abundancia y diversidad de los ectoparásitos, así como la relación filogenética con sus hospederos es esencial para profundizar en el estudio de las asociaciones hospedero-parásito.

Palabras clave: Abundancia, biomonitoreo, diversidad, fragmentación, riqueza.

ABSTRACT. The influence of the anthropogenic transformation of the landscape by livestock activities on the composition and specificity of ectoparasite communities associated with bats was evaluated in livestock productions of the state of Veracruz. Sites with different levels of landscape transformation were compared, including productions under grazing systems in fallow land, silvopasture, and grassland. A total of 944 bats of 23 species were captured in eight livestock production units. The taxonomic identification of 1433 ectoparasites of 35 species obtained from 428 hosts was carried out. Analyses of taxonomic diversity and phylogenetic specificity were performed between the bat-ectoparasite associations. The composition of the ectoparasite communities associated with Artibeus jamaicensis showed significant differences between the three levels of landscape transformation. The acahual (fallow land), which is the landscape with the least anthropogenic transformation, presented the highest values of diversity of bat species and ectoparasites. The phylospecificity of the streblid bat flies did not show significant changes between the different types of landscape in the study. The consideration of anthropogenic factors that affect the richness, abundance and diversity of the ectoparasites, as well as the phylogenetic relationship with their hosts, is essential to achieve an in-depth study of host-parasite associations.

Key words: Abundance, biomonitoring, diversity, fragmentation, richness. 


\section{INTRODUCCIÓN}

La fragmentación antropogénica del hábitat altera la geometría espacial, la conectividad y el grado de aislamiento de los fragmentos remanentes. EI cambio de uso de suelo asociado a estas modificaciones afecta los procesos ecológicos a diferentes niveles, desde el comportamiento de los individuos y la dinámica de las poblaciones, hasta la composición y estructura de las comunidades y los flujos de materia y energía (Trilleras et al. 2015). Los bosques tropicales albergan el $70 \%$ de la biodiversidad mundial. Pero se estima que en la región tropical se pierde aproximadamente 15 millones de hectáreas de bosque maduro por año, de las cuales el $60 \%$ corresponden a la fragmentación derivada de prácticas agrícolas, pecuarias y forestales, urbanización, minería, tala furtiva e incendios (Cristóbal et al. 2014).

Entender cómo se modifican los patrones ecológicos en hábitats fragmentados es clave para comprender el impacto que tiene la perturbación antropogénica sobre la dinámica de los ecosistemas $y$ escalas (Ries et al. 2004). Por ello es fundamental evaluar los cambios en la estructura de las comunidades y, en consecuencia, en la red de interacciones entre las diferentes especies que las componen (Suzán et al. 2008). El uso de bioindicadores es una herramienta útil para conocer el grado de integridad de un ecosistema mediante el monitoreo de especies de vertebrados e invertebrados con altas abundancias, y que además sean ecológica, taxonómica y tróficamente diversas. Las especies bioindicadoras tienen un papel importante dentro del ecosistema y responden de forma cuantitativa y predecible a cambios en el entorno, por lo que el análisis de métricas ecológicas como abundancia y diversidad de especies puede ayudar a detectar cambios ambientales (Russo y Jones 2015).

Los murciélagos son el segundo orden de mamíferos con mayor diversidad de especies en el mundo, comprendiendo más de 1300 especies (Voigt y Kingston 2016). En México este orden está conformado por 140 especies, de las cuales 16 son endémicas (Ceballos y Arroyo-Cabrales 2012,
Ramírez-Pulido et al. 2014). La elevada dependencia que presentan algunas especies a hábitats específicos, el grado de especialización en su alimentación y el uso de refugios, las hace susceptibles a las modificaciones del paisaje, por lo que han sido propuestas como bioindicadores del estado de conservación de bosques húmedos neotropicales (Medellín et al. 2000, Russo y Jones 2015). La importancia de este grupo de mamíferos se debe, entre otros factores, a la diversidad de procesos ecológicos en los que interviene, como la polinización de plantas y dispersión de semillas que son importantes para la regeneración de selvas y bosques tropicales, además de desempeñarse como control biológico de poblaciones de insectos (Kunz et al. 2011).

El interés por el estudio del papel de los murciélagos como hospederos de una gran diversidad de endoparásitos, ectoparásitos y diversos agentes patógenos ha aumentado en los últimos años. Entre los ectoparásitos asociados a los murciélagos en América se han registrado principalmente a las familias Nycteribiidae, Streblidae, Spinturnicidae, Spelaeorhynchidae, Ixodidae, Argasidae e Ischnopsyllidae (Colín-Martínez y García-Estrada 2018, Colín-Martínez et al. 2018, Cuxim-Koyoc et al. 2018, Czenze y Broders 2011, González-Acuña et al. 2018). Los ácaros pertenecientes a las familias Spinturnicidae y Spelaeorhynchidae, específicos de murciélagos, presentan adaptaciones que responden a una forma de vida ectoparasitaria que incluyen hábitos hematófagos. Los dípteros de las familias Nycteribiidae y Streblidae son los ectoparásitos más abundantes y mejor adaptados de los murciélagos (Dittmar et al. 2006).

En distintos estudios, el hospedero suele considerarse como hábitat para el parásito, ya que el cuerpo del hospedero proporciona una amplia gama de estímulos ambientales y recursos y juega un papel importante en la configuración de la historia de vida, la ecología y el comportamiento de los parásitos (Presley 2010). Sin embargo, el nivel de infestación de un hospedero por un ectoparásito particular depende no sólo de las características del hospedero, sino también del hábitat y del tipo de refugio del murciélago (Patterson et al. 2007, Pilosof et al. 2012). 
Las condiciones ambientales afectan la dinámica de las poblaciones de parásitos, así como los ensambles e interacciones a nivel de comunidad entre parásitos y hospederos. Distintas especies de parásitos que son sensibles a las modificaciones del hábitat del hospedero han sido reconocidas como posibles bioindicadores (Mackenzie y Hemmingsen 2014, Patterson et al. 2007, Patterson et al. 2008).

En trabajos recientes se ha estudiado el efecto de la perturbación antropogénica sobre la prevalencia de ectoparásitos de murciélagos orientados a las interacciones entre el ambiente, el hospedero y los parásitos. La modificación de estas interacciones puede desencadenar procesos de selección en los ectoparásitos, modificando la especificidad filogenética hacia sus hospederos por ausencia o presencia de estos (Dittmar et al. 2015). A su vez incrementa la probabilidad de encontrar hospederos accidentales en sitios con distintos grados de perturbación (Poulin et al. 2011). Por ello, resulta necesario considerar las condiciones ambientales, las variables climáticas, la presencia de actividades antropogénicas y la fragmentación de los hábitats en el estudio de las comunidades de ectoparásitos (Bolivar-Cimé et al. 2018, Hernández-Martínez et al. 2018). El objetivo del presente trabajo fue evaluar la composición y la filoespecificidad de comunidades de ectoparásitos asociados a murciélagos en tres tipos de paisajes agropecuarios en el estado de Veracruz.

\section{MATERIALES Y MÉTODOS}

\section{Descripción del área de estudio}

El trabajo se desarrolló en el centro y sur del estado de Veracruz. La ubicación geográfica del estado ofrece un entorno de características tropicales, que se modifican por la influencia de las cadenas montañosas con climas que varían de muy calientes a muy fríos. La precipitación anual oscila entre 500 $\mathrm{mm}$ en algunos lugares del norte y alrededor de 4 $000 \mathrm{~mm}$ en el sur (Estrada-Contreras et al. 2015). Se distinguen dos épocas: 1) secas, de marzo a mayo con precipitación menor a $100 \mathrm{~mm}$ por mes, y 2) lluvias, de junio a febrero con precipitaciones superiores a $200 \mathrm{~mm}$ por mes.

\section{Sitios de estudio}

Los ocho sitios de muestreo se ubican en los municipios de Tlapacoyan, Martínez de la Torre, Úrsulo Galván, Puente Nacional y San Andrés Tuxtla. Cada sitio corresponde a una unidad de producción pecuaria con bovinos de doble propósito y la distancia mínima entre cada uno fue de al menos 2 $\mathrm{km}$. Las unidades de producción, que en general se encuentran establecidas bajo un sistema ganadero de pastoreo controlado, representan un gradiente de paisajes con diferente nivel de transformación (Tabla 1). Para fines de este estudio, se consideraron tres categorías de paisaje: pastizal, silvopastoril y acahual. Se consideró como pastizal inducido a aquellos sitios cuyo paisaje estuviera dominado por la presencia de gramíneas forrajeras; silvopastoril a los sitios en los que una combinación de gramíneas forrajeras, leguminosas arbóreas y arbustivas dominara el paisaje; y acahual a los sitios con paisajes dominados por vegetación secundaria derivada de una selva alta perennifolia que incluyera la presencia de árboles, arbustos, herbáceas y gramíneas.

\section{Captura de murciélagos}

El trabajo de campo se realizó de septiembre a noviembre de 2016, meses que corresponden a la época de lluvias, y de marzo a mayo de 2017, en la época de secas. Para capturar los murciélagos se utilizaron ocho redes de niebla de $12 \mathrm{~m}$ de largo por $2.5 \mathrm{~m}$ de altura en cada sitio de muestreo por noche. Por cada sitio, se muestreó durante dos noches en lluvias y dos noches en secas, con un esfuerzo de muestreo de $7 \mathrm{~h} /$ red por noche para un total de $28 \mathrm{~h} / \mathrm{red}$ por sitio de muestreo considerando ambas temporadas. La colocación de las redes fue pareada y la distancia mínima entre cada grupo de redes fue de 50 metros para evitar competencia entre ellas. Cada red se revisó con un periodo no mayor a 30 minutos entre una liberación y otra para evitar perturbar el área de captura y minimizar el tiempo en que los murciélagos quedaran enmallados en la red. Una vez capturado, cada individuo fue man- 
Tabla 1. Sitios de muestreo y coordenadas geográficas.

\begin{tabular}{ccccc}
\hline Sitio & Paisaje & Municipio & \multicolumn{2}{c}{ Coordenadas } \\
\hline Clarín & Pastizal 1 & Tlapacoyan & $20^{\circ} 0203$ Norte & $97^{\circ} 0614$ Oeste \\
Soledad & Silvopastoril 1 & Martínez de la Torre & $20^{\circ} 0208$ Norte & $97^{\circ} 0439$ Oeste \\
Encinar & Acahual 1 & Tlapacoyan & $19^{\circ} 5443$ Norte & $97^{\circ} 1210$ Oeste \\
CBTA 17 & Silvopastoril 2 & Úrsulo Galván & $19^{\circ} 2434$ Norte & $96^{\circ} 2233$ Oeste \\
Utrera & Acahual 2 & Puente Nacional & $19^{\circ} 1706$ Norte & $96^{\circ} 3111$ Oeste \\
Perico & Pastizal 2 & San Andrés Tuxtla & $18^{\circ} 3942$ Norte & $95^{\circ} 1255$ Oeste \\
Divino & Pastizal 3 & San Andrés Tuxtla & $18^{\circ} 3832$ Norte & $95^{\circ} 1136$ Oeste \\
Ruiz & Acahual 3 & San Andrés Tuxtla & $18^{\circ} 4013$ Norte & $95^{\circ} 0734$ Oeste \\
\hline
\end{tabular}

tenido dentro de un saco de tela de manera individual para posteriormente obtener los valores morfométricos necesarios para su identificación taxonómica. La identificación hasta nivel de especie se realizó con la guía de campo de Medellín et al. (2008). La captura y manejo de los organismos se realizó cumpliendo las directrices de la Sociedad Americana de Mastozoología para el uso de mamíferos silvestres en investigación (Sikes y Bryan 2016).

\section{Captura e identificación ectoparásitos}

Los murciélagos capturados se sometieron a inspección visual sistematizada, en la que se capturaron todos los ectoparásitos que se detectaron a simple vista. Para localizar los ectoparásitos, la búsqueda se realizó de la cabeza a los miembros pélvicos con énfasis en regiones como orejas, propatagio, plagiopatagio y uropatagio. La captura de ectoparásitos se llevó a cabo con la ayuda de pinzas entomológicas en los murciélagos. Una vez obtenida la muestra se liberó a cada individuo. Las muestras se colocaron en tubos de $2 \mathrm{ml}$ con aproximadamente $1.5 \mathrm{ml}$ de alcohol al $70 \%$ para fijarlos y se mantuvieron a temperatura ambiente (Bezerra et al. 2016). En el laboratorio, los ácaros presentes en las muestras se separaron para someterlos a un proceso de aclarado con lactofenol a temperatura ambiente (de Almeida et al. 2017) por aproximadamente 45 días. Una vez aclarados se retiraron de los tubos y de manera directa se realizó el montaje en medio CMC-10. Todas las muestras montadas se colocaron en charolas de metal y se realizó la desecación del medio con ayuda de una incubadora a $50{ }^{\circ} \mathrm{C}$ durante una semana (Kazak et al. 2017). Para una mejor conservación de los montajes se realizó el sellado de los cubreobjetos con un anticorrosivo formulado a base de resinas alquidálicas y cromato de zinc. Para la identificación taxonómica de los ectoparásitos se utilizaron microscopios estereoscópico y óptico (Wenzel 1976).

\section{Análisis de datos}

Todos los análisis se realizaron con la ayuda del software libre $R$ v. 3.4.3. La descripción de la diversidad de especies de hospederos y ectoparásitos se realizó con el índice de entropía de Shannon. Con los valores de diversidad de hospederos y ectoparásitos se calculó el coeficiente de correlación de Pearson. Se generaron curvas de rango-abundancia para describir la estructura de la comunidad de especies de ectoparásitos por sitio de muestreo y por especie hospedera. A la variable de la abundancia relativa se le realizó la prueba de Shapiro-Wilk para conocer la distribución de los datos. Se realizó un análisis de covarianza (ANCOVA) considerando en nuestro modelo como variable respuesta a la abundancia relativa de los ectoparásitos, como variable explicativa al tipo de paisaje y como covariable al rango de las especies ectoparásitas (Abella-Medrano et al. 2015). La diversidad beta de las comunidades de ectoparásitos y hospederos de los ocho sitios se cuantificó con el índice de Morisita-Horn. Se utilizó el coeficiente de correlación de Pearson para analizar la relación entre las abundancias relativas de todos los pares de especies ectoparásitas de las especies de murciélagos generalistas (López-Pérez et al. 2018).

Para determinar la especificidad filogenética de los ectoparásitos, es decir el grado de asociación parásito-hospedero explicado por la filogenia de los hospederos, se comparó el efecto de la diversidad filogenética, entendido como el agrupamiento filogenético (AF) de las especies de murciélagos uti- 
lizadas por una especie parásita determinada contra un modelo nulo basado en el efecto del tamaño estandarizado de la muestra total (Poulin et al. 2011). El árbol filogenético utilizado como base para realizar el cálculo de AF fue el súper-árbol filogenético de mamíferos publicado por Bininda-Emonds (2007).

\section{RESULTADOS}

Se registraron murciélagos de las familias Phyllostomidae y Mormoopidae, con un total de 23 especies, de las cuales las más abundantes fueron Artibeus jamaicensis, A. lituratus y Sturnira hondurensis. Dichas especies estuvieron presentes en todos los sitios de muestreo. Se capturaron 944 individuos, de los cuales 428 fueron portadores de al menos un ectoparásito; la prevalencia total de ectoparásitos fue de 45.34\% (IC 42.16 - 48.51\%). Se realizó la identificación taxonómica de 1433 ectoparásitos, 995 dípteros de la familia Streblidae, 432 ácaros de la familia Spinturnicidae y seis ácaros de la familia Spelaeorhynchidae, para un total de 35 especies. Las especies más abundantes fueron Trichobius costalimai (Streblidae) y Periglischrus iheringi (Spinturnicidae). Para los estréblidos, se registraron especies de las tres subfamilias registradas para el Nuevo Mundo. La riqueza de ectoparásitos fue de 32 especies para la familia Phyllostomidae y cuatro para la familia Mormoopidae; entre ambas familias se compartió el registro de Periglischrus ojastii (Tabla 2). Las especies hospederas con mayor número de asociaciones de especies ectoparásitas fueron $A$. jamaicensis, $A$. lituratus y $S$. hondurensis.

El esfuerzo de muestreo de ectoparásitos representó una cobertura de la riqueza estimada de $99.5 \%$ (35 especies), por lo que la curva de acumulación de especies de ectoparásitos tendió a alcanzar la asíntota (Figura 1). El sitio con los valores de riqueza más altos, tanto en especies de murciélagos como de ectoparásitos, fue el Acahual 3, además de ser el sitio con mayor diversidad de hospederos. El sitio con el valor de diversidad de especies de ectoparásitos más alto fue el Acahual 1
(Tabla 3). El acahual fue el tipo de paisaje que obtuvo los valores más altos de diversidad de especies de murciélagos y ectoparásitos. Cabe resaltar que el pastizal, que es el paisaje con mayor transformación antropogénica, presentó valores más altos de diversidad de hospederos y ectoparásitos en comparación con el paisaje silvopastoril. A través del cálculo del coeficiente de correlación de Pearson, se detectó una relación positiva, marginalmente significativa $(r=$ $0.67, p=0.06$ ), entre los valores de diversidad de especies de hospederos y de diversidad de especies de ectoparásitos por sitio de muestreo.

En el paisaje de tipo pastizal se registraron 17 especies de ectoparásitos, de las cuales Paratrichobius dunni fue exclusiva para este paisaje. Para el paisaje de tipo silvopastoril se registraron 15 especies, dos de ellas exclusivas: Nycterophilia mormoopsis y $P$. lowei. Para el paisaje de tipo acahual se registraron un total de 11 especies exclusivas y presentó el valor más alto de riqueza de especies ectoparásitas (31 especies; Tabla 2). De todas las especies hospederas, únicamente $A$. jamaicensis, $A$. lituratus, $S$. hondurensis y $S$. parvidens fueron capturados en todos los sitios de muestreo. Con base en los valores de abundancia relativa, se consideró a $A$. jamaicensis, A. lituratus y $S$. hondurensis como las especies mejor representadas en el muestreo (Figura 1). A. jamaicensis obtuvo los valores más altos de diversidad de especies de ectoparásitos, seguido por $S$. hondurensis y en tercer lugar $A$. lituratus. La comunidad de ectoparásitos para los 209 hospederos de la especie $A$. jamaicensis estuvo integrada por 645 ectoparásitos, de tres familias: Streblidae (10 especies), Spinturnicidae (dos especies), Spelaeorhynchidae (una especie), con la mayor riqueza de ectoparásitos asociados. Las especies ectoparásitas más abundantes en los tres tipos de paisaje fueron Periglischrus iheringi (Spinturnicidae), que dominó en acahual y pastizal, Trichobius assimilis (Streblidae) y Megistopoda aranea (Streblidae), esta última la más abundante en silvopastoril. En el caso de la composición de la comunidad de ectoparásitos asociados con $A$. lituratus, de los 66 hospederos parasitados se obtuvieron 142 ejemplares pertenecientes a dos familias: Streblidae 
Orta-Pineda et al.

Tabla 2. Número de individuos capturados de cada especie de ectoparásito por especie hospedera y por tipo de paisaje.

\begin{tabular}{|c|c|c|c|c|}
\hline \multirow[t]{2}{*}{ Ectoparásito } & \multicolumn{3}{|c|}{ ATE } & \multirow[t]{2}{*}{ Hospedero } \\
\hline & $\mathrm{P}$ & S & A & \\
\hline \multirow[t]{3}{*}{ Anastrebla mattadeni } & 0 & 0 & 1 & Artibeus jamaicensis \\
\hline & 0 & 0 & 1 & Carollia perspicillata \\
\hline & 0 & 0 & 1 & Glossophaga soricina \\
\hline \multirow[t]{2}{*}{ Aspidoptera delatorrei } & 0 & 6 & 0 & Sturnira hondurensis \\
\hline & 2 & 5 & 0 & Sturnira parvidens \\
\hline \multirow[t]{3}{*}{ Aspidoptera falcata } & 0 & 2 & 0 & Artibeus lituratus \\
\hline & 1 & 1 & 6 & Sturnira hondurensis \\
\hline & 0 & 0 & 2 & Sturnira parvidens \\
\hline \multirow[t]{5}{*}{ Aspidoptera phyllostomatis } & 56 & 4 & 39 & Artibeus jamaicensis \\
\hline & 0 & 0 & 2 & Phyllostomus discolor \\
\hline & 0 & 0 & 1 & Platyrrhinus helleri \\
\hline & 1 & 2 & 1 & Sturnira hondurensis \\
\hline & 0 & 1 & 1 & Sturnira parvidens \\
\hline \multirow[t]{5}{*}{ Megistopoda aranea } & 34 & 23 & 100 & Artibeus jamaicensis \\
\hline & 0 & 4 & 1 & Artibeus lituratus \\
\hline & 0 & 0 & 1 & Phyllostomus discolor \\
\hline & 0 & 0 & 1 & Platyrrhinus helleri \\
\hline & 2 & 0 & 3 & Sturnira hondurensis \\
\hline \multirow[t]{4}{*}{ Megistopoda próxima } & 0 & 0 & 10 & Artibeus jamaicensis \\
\hline & 0 & 0 & 1 & Glossophaga commissarisi \\
\hline & 4 & 8 & 29 & Sturnira hondurensis \\
\hline & 5 & 6 & 8 & Sturnira parvidens \\
\hline Metelasmus pseudopterus & 1 & 0 & 2 & Artibeus jamaicensis \\
\hline \multirow[t]{2}{*}{ Nycterophilia coxata } & 0 & 0 & 2 & Artibeus jamaicensis \\
\hline & 0 & 0 & 1 & Leptonycteris yerbabuenae \\
\hline Nycterophilia mormoopsis & 0 & 1 & 0 & Mormoops megalophylla \\
\hline Paratrichobius dunni & 2 & 0 & 0 & Dermanura tolteca \\
\hline \multirow[t]{3}{*}{ Paratrichobius longicrus } & 0 & 2 & 1 & Artibeus jamaicensis \\
\hline & 12 & 27 & 6 & Artibeus lituratus \\
\hline & 0 & 1 & 4 & Sturnira hondurensis \\
\hline Paratrichobius lowei & 0 & 1 & 0 & Artibeus lituratus \\
\hline Paratrichobius salvini & 1 & 0 & 1 & Platyrrhinus helleri \\
\hline \multirow[t]{2}{*}{ Paratrichobius sanchezi } & 3 & 0 & 5 & Platyrrhinus helleri \\
\hline & 0 & 0 & 1 & Uroderma bilobatum \\
\hline \multirow[t]{2}{*}{ Periglischrus caligus } & 0 & 0 & 5 & Glossophaga commissarisi \\
\hline & 0 & 0 & 5 & Glossophaga soricina \\
\hline \multirow[t]{3}{*}{ Periglischrus herrerai } & 0 & 0 & 4 & Artibeus jamaicensis \\
\hline & 0 & 1 & 1 & Artibeus lituratus \\
\hline & 0 & 0 & 1 & Sturnira parvidens \\
\hline \multirow[t]{6}{*}{ Periglischrus iheringi } & 132 & 15 & 132 & Artibeus jamaicensis \\
\hline & 16 & 42 & 18 & Artibeus lituratus \\
\hline & 7 & 0 & 0 & Dermanura tolteca \\
\hline & 0 & 0 & 1 & Dermanura watsoni \\
\hline & 1 & 0 & 4 & Platyrrhinus helleri \\
\hline & 4 & 0 & 0 & Uroderma bilobatum \\
\hline \multirow[t]{3}{*}{ Periglischrus ojastii } & 0 & 0 & 1 & Pteronotus parnellii \\
\hline & 0 & 1 & 8 & Sturnira hondurensis \\
\hline & 0 & 4 & 4 & Sturnira parvidens \\
\hline Periglischrus torrealbai & 0 & 0 & 25 & Phyllostomus discolor \\
\hline \multirow[t]{2}{*}{ Speiseria ambigua } & 0 & 0 & 4 & Carollia perspicillata \\
\hline & 0 & 0 & 1 & Carollia sowelli \\
\hline Spelaeorhynchus praecursor & 0 & 0 & 4 & Artibeus jamaicensis \\
\hline & 0 & 0 & 2 & Carollia perspicillata \\
\hline Strebla harderi & 0 & 0 & 1 & Carollia perspicillata \\
\hline Strebla hertigi & 0 & 0 & 1 & Artibeus jamaicensis \\
\hline & 0 & 0 & 2 & Carollia sowelli \\
\hline & 0 & 0 & 9 & Phyllostomus discolor \\
\hline
\end{tabular}


Tabla 2. Continuación.

\begin{tabular}{ccccc}
\hline Ectoparásito & ATE & & Hospedero \\
& P & S & A & \\
\hline Strebla wiedemanni & 2 & 0 & 13 & Desmodus rotundus \\
Trichobioides perspicillatus & 0 & 0 & 54 & Phyllostomus discolor \\
Trichobius assimilis & 17 & 9 & 54 & Artibeus jamaicensis \\
& 6 & 5 & 0 & Artibeus lituratus \\
Trichobius caecus & 2 & 0 & 0 & Sturnira hondurensis \\
Trichobius costalimai & 3 & 0 & 8 & Pteronotus parnelli \\
Trichobius galei & 0 & 0 & 187 & Phyllostomus discolor \\
Trichobius handleyi & 0 & 0 & 1 & Pteronotus davyi \\
& 0 & 0 & 6 & Glossophaga commissarisi \\
Trichobius joblingi & 0 & 3 & 2 & Glossophaga soricina \\
& 5 & 0 & 18 & Carollia perspicillata \\
Trichobius keenani & 0 & 0 & 9 & Carollia sowelli \\
& 0 & 0 & 3 & Glossophaga commissarisi \\
Trichobius parasiticus & 1 & 2 & 15 & Glossopha soricina \\
Trichobius sphaeronotus & 6 & 0 & 91 & Desmodus rotundus \\
Trichobius tuttlei & 0 & 0 & 2 & Leptonycteris yerbabuenae \\
& 0 & 1 & 1 & Artibeus jamaicensis \\
Total & 0 & 0 & 1 & Phyllostomus discolor \\
\hline
\end{tabular}

$\overline{(A T E}=$ Abundancia total de ectoparásitos, $\mathrm{P}=$ Pastizal, $\mathrm{S}=$ Silvopastoril, $\mathrm{A}=\mathrm{Ac}-$ ahual)

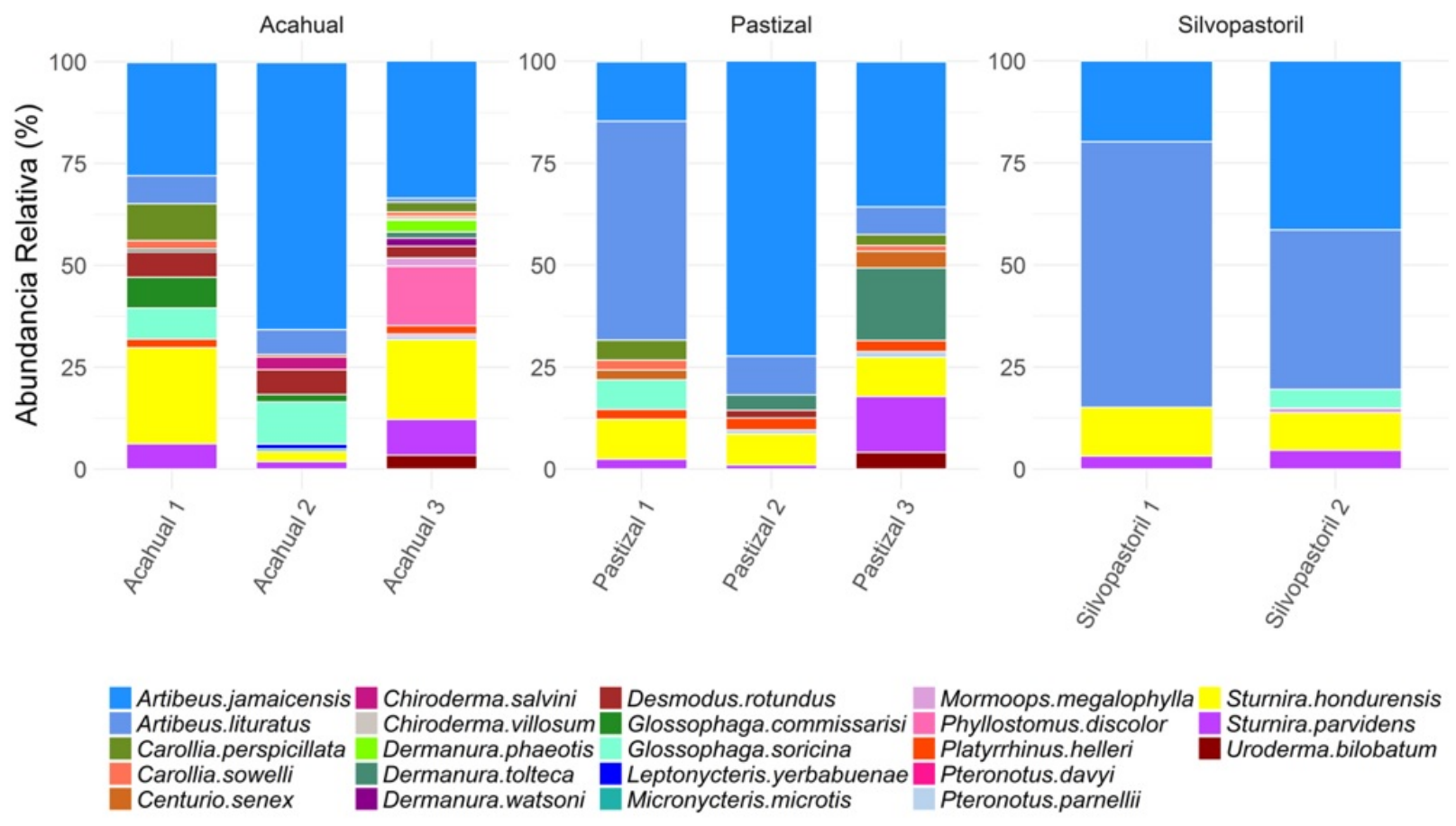

Figura 1. Composición de las comunidades de murciélagos por sitio de muestreo.

(cinco especies) y Spinturnicidae (dos especies). Las especies más abundantes para los tres tipos de paisaje fueron Periglischrus iheringi (Spinturnicidae) y Megistopoda aranea (Streblidae). La comunidad de ectoparásitos de los 39 hospederos de la especie
S. hondurensis estuvo compuesta por 80 ectoparásitos de dos familias: Streblidae (siete especies) y Spinturnicidae (una especie). Megistopoda proxima (Streblidae) fue la más abundante en los tres tipos de paisaje. La composición de las comunidades de ec- 
Tabla 3. Análisis de la diversidad de hospederos y ectoparásitos por tipo de paisaje.

\begin{tabular}{|c|c|c|c|c|}
\hline \multirow[t]{2}{*}{ Sitio } & \multicolumn{2}{|c|}{ Riqueza de especies } & \multicolumn{2}{|c|}{ Diversidad de especies (Shannon) } \\
\hline & Murciélagos & Ectoparásitos & Murciélagos & Ectoparásitos \\
\hline Pastizal 1 & 9 & 7 & 1.54 & 1.57 \\
\hline Pastizal 2 & 8 & 10 & 1.04 & 1.36 \\
\hline Pastizal 3 & 11 & 12 & 1.93 & 1.89 \\
\hline Silvopastoril 1 & 4 & 10 & 0.96 & 1.92 \\
\hline Silvopastoril 2 & 6 & 11 & 1.28 & 1.93 \\
\hline Acahual 1 & 11 & 20 & 2.03 & 2.45 \\
\hline Acahual 2 & 11 & 16 & 1.31 & 1.81 \\
\hline Acahual 3 & 18 & 23 & 2.09 & 2.24 \\
\hline
\end{tabular}

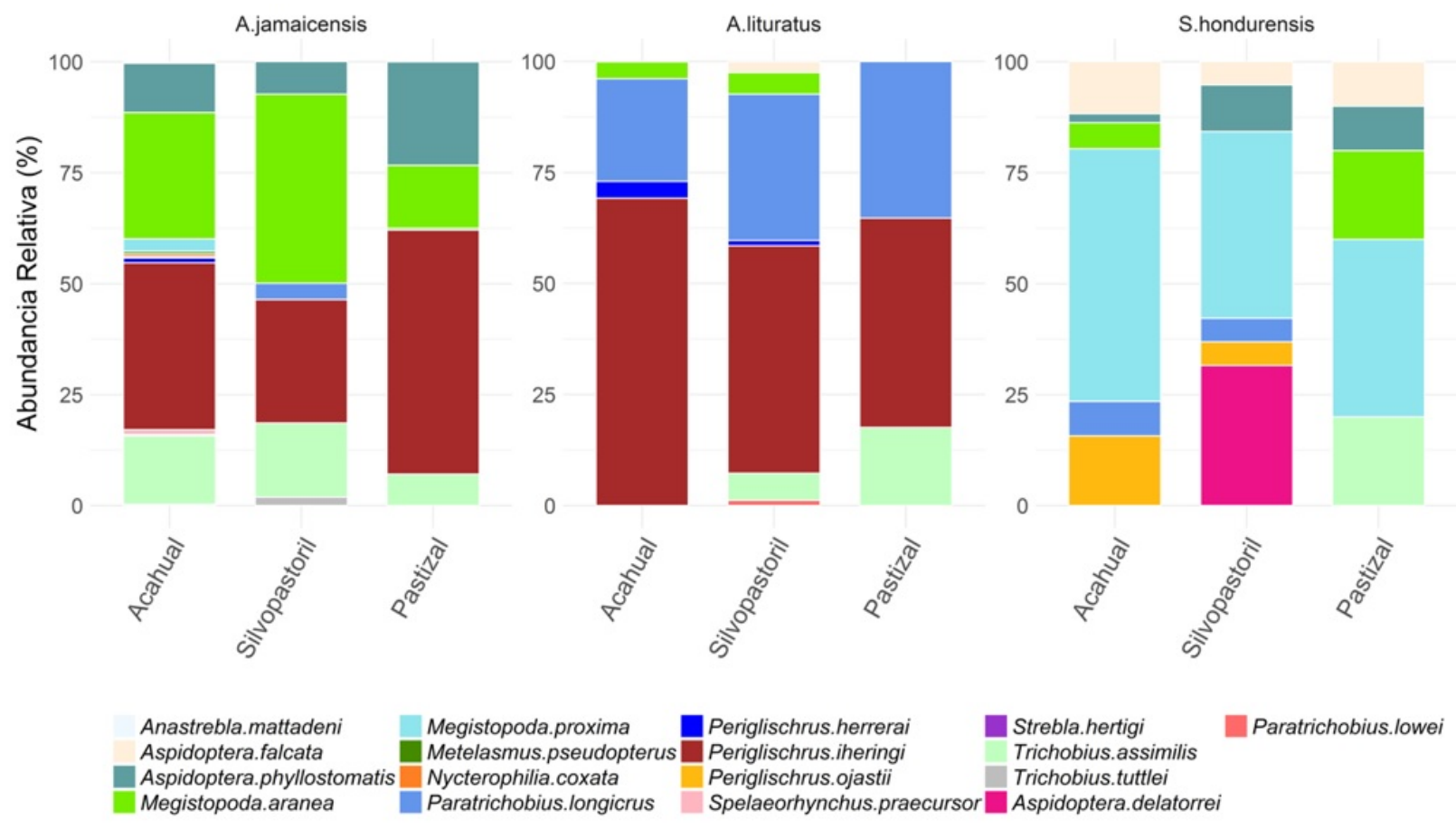

Figura 2. Composición de las comunidades de ectoparásitos de los hospederos generalistas de hábitat.

toparásitos en los hospederos generalistas de hábitat se muestra en la Figura 2.

Para el ANCOVA sólo se consideró a $A$. jamaicensis como especie hospedera, debido a que fue la única que presentó más de una especie de ectoparásito en cada sitio de muestreo. La interacción entre el tipo de paisaje y el rango de las especies ectoparásitas demostró un efecto estadísticamente significativo sobre la abundancia relativa de los ectoparásitos $(F=7.034$ y $p=0.005)$. Los resultados de la diversidad beta (Morisita-Horn) entre las comunidades de ectoparásitos (por tipo de paisaje) muestran en general una alta similitud para cada es- pecie hospedera generalista. Las comunidades de ectoparásitos de $S$. hondurensis presentaron valores altos de similitud de acuerdo con su composición, agrupándose en la misma rama, sin importar el tipo de paisaje (Figura 3). En cuanto a la relación entre las abundancias relativas de especies ectoparásitas con las especies hospederas generalistas de hábitat, la más fuerte fue una relación negativa entre la abundancia relativa de Paratrichobius longicrus y la abundancia relativa de Periglischrus iheringi en $A$. lituratus $(r=-0.64$ y $p<0.0001)$.

La comparación de los valores de AF de las siete especies de estréblidos representados en to- 


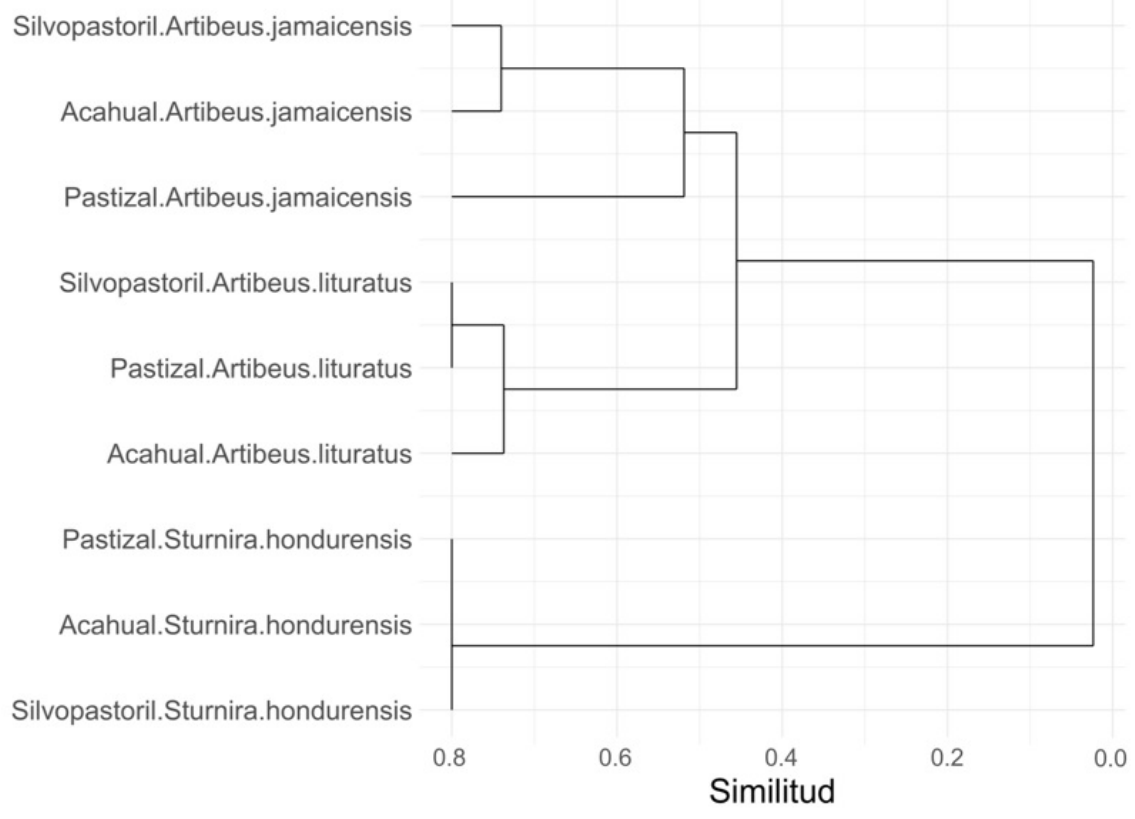

Figura 3. Dendograma de similitud de Morisita-Horn de las comunidades de ectoparásitos por tipo de paisaje de los tres hospederos generalistas (A. jamaicensis, A. lituratus y S. hondurensis).

dos los tipos de paisaje. Los valores de AF calculados por frecuencia muestran que la mayoría de los ectoparásitos tienen una elevada asociación filogenética. Sin embargo, los valores de AF fueron similares en los tres tipos de paisaje, es decir, no se observó diferencia significativa entre las medias de los valores de AF en estas siete especies de ectoparásitos de murciélagos muestreados en diferentes tipos de paisaje. A excepción de Trichobius assimilis cuyo valor de AF mostró un patrón de asociación filogenética significativamente distinto entre acahual y los sitios silvopastoril y pastizal.

\section{DISCUSIÓN}

En las últimas cuatro décadas, los ecosistemas del estado de Veracruz han sido modificados sustancialmente por las actividades antropogénicas, entre las que destaca la ganadería por su amplia distribución y por el grado de transformación que imprime sobre los paisajes naturales a través del establecimiento de potreros y zonas de cultivo. Estas modificaciones en la estructura del paisaje no sólo impactan las características de la vegetación, sino que, además alteran la dinámica de las comunidades de fauna, tanto de vertebrados como de invertebrados. Por lo tanto, los cambios en la composición de las comunidades reflejan el efecto que tiene la modificación de la estructura del paisaje en regiones con alta actividad pecuaria (Gonçalves et al. 2017, Newbold et al. 2015).

Si bien la relación positiva entre la diversidad de hospederos y la diversidad de ectoparásitos resulta lógica al considerar que conforme aumenta la captura de especies de murciélagos, se incrementa la probabilidad de acumular más hospederos y, por ende, mayor cantidad de especies ectoparásitas (Krasnov et al. 2004), es importante estudiar los efectos de la composición de las comunidades de especies hospederas sobre la composición de las comunidades de ectoparásitos asociados. La composición de las comunidades de especies hospederas en paisajes transformados, junto con sus patrones de distribución y de asociación con ciertos parásitos, ha sido ampliamente investigada. Sin embargo, existen pocos trabajos que presten atención a la composición, a la especificidad filogenética y a los patrones de organización de las comunidades de parásitos, es- 
pecialmente las de ectoparásitos en murciélagos, en paisajes modificados por el hombre (Tello et al. 2008).

Las comunidades de hospederos y parásitos son sensibles a las condiciones del ambiente externo, incluida la perturbación antropogénica (Pilosof et al. 2012). Por ello, variables como la abundancia, riqueza, diversidad y el grado de infestación de los parásitos, pueden responder a cambios en el tamaño de las colonias, la masa corporal de los hospederos y factores asociados a la fragmentación del hábitat. Sin embargo, sólo se ha demostrado el efecto de la fragmentación del hábitat sobre la riqueza de especies de helmintos y virus asociados a murciélagos (Gay et al. 2014, Rico-Chavez et al. 2015).

El análisis de la diversidad y riqueza de las comunidades de ectoparásitos muestra que los valores más altos se registraron en sitios conformados principalmente por acahual. Los acahuales suelen estar asociados a paisajes más heterogéneos que se caracterizan por la presencia de vegetación secundaria, fragmentos remanentes de bosque maduro y zonas de cultivo o potreros (Pérez-Nicolás et al. 2018). Al respecto Froeschke et al. (2013) encontraron que la riqueza de especies de ectoparásitos fue más alta en sitios fragmentados que en sitios conservados, atribuyendo este patrón a la heterogeneidad del paisaje. Pero las comunidades de ectoparásitos en nuestro trabajo están compuestas en gran medida por las mismas especies independientemente de las características del paisaje. De manera similar, Frank et al. (2016), reportan que los ensambles de ectoparásitos en las comunidades de murciélagos de su estudio fueron muy similares en composición entre los sitios que muestrearon, los cuales presentan diferente grado de deforestación. Se ha demostrado que la composición del paisaje tiene un gran peso en el valor ecológico del mismo ya que provee de diversos recursos, como cobertura y alimento a las especies que transitan entre los pastizales, ayudando a mantener la diversidad del área (Daily et al. 2014).

Las especies ectoparásitas Periglischrus iheringi, Trichobius assimilis, Trichobius tuttlei, Aspidoptera phyllostomatis y Megistopoda aranea fueron compartidas entre los tres diferentes tipos de paisaje agropecuario del estudio. Dado que las especies de murciélagos generalistas de hábitat dominan en paisajes fragmentados, la estructura de la comunidad de los ectoparásitos asociados a estos sitios reflejó tal dominancia. Sin embargo, las comunidades de ectoparásitos de las tres especies de murciélagos generalistas de hábitat no mostraron diferencias significativas en cuanto a su composición por tipo de paisaje y únicamente se observó una variabilidad en cuanto a las abundancias relativas de las especies ectoparásitas. De manera similar, Frank et al. (2016) determinaron que la cobertura forestal no es un predictor significativo para el análisis de la abundancia y la composición de las especies ectoparásitas asociadas a murciélagos.

En estudios realizados en Chiapas y Yucatán se reportaron valores de prevalencia de ectoparásitos por sitio entre 11 y $85 \%$ (Cuxim-Koyoc et al. 2018, Tlapaya-Romero et al. 2015). La prevalencia de murciélagos parasitados en el presente trabajo fue del $45.34 \%$. Estas diferencias pueden estar relacionadas con las condiciones ambientales de cada localidad (temperatura, humedad, altitud y precipitación), el esfuerzo de muestreo (específicamente la cantidad de murciélagos capturados) y las variaciones en las comunidades de hospederos (Lourenço et al. 2016). Por otro lado, Patterson et al. (2007) sugieren que los hábitos de refugio pueden impactar significativamente en los niveles de parasitismo. La prevalencia, la abundancia y la riqueza de especies de ectoparásitos en los hospederos aumenta en especies hospederas que forman colonias de muchos individuos y que se refugian en sitios que mantienen la temperatura y humedad ideales para completar el ciclo de vida de los parásitos, como es el caso de las especies de murciélagos que se refugian en cuevas. En el presente estudio, las especies de murciélagos Centurio senex, Chiroderma salvini, Chiroderma villosum, Dermanura phaeotis y Micronycteris microtis no presentaron ectoparásitos. Estos casos, en los que no existen registros de ectoparasitismo, pueden deberse a los hábitos de cada especie, por ejemplo, el constante recambio del refugio o simplemente el pequeño tamaño de sus colonias.

Algunos estudios en murciélagos han descrito relaciones negativas entre diferentes especies de ec- 
toparásitos a través del análisis de la correlación entre sus densidades (Tello et al. 2008). En el estudio se encontró fuerte relación negativa entre la abundancia relativa de los ectoparásitos Paratrichobius longicrus (Trichobiinae) y Periglischrus iheringi (Spinturnicidae) en la especie hospedera Artibeus lituratus. Esta relación podría ser el resultado de interacciones competitivas entre estas especies, ya que ambas tienden a ocupar las superficies del hospedero desprovistas de pelo. Este tipo de interacciones podría presentarse entre otras especies, pues se ha documentado que las especies de ectoparásitos tienen preferencia por zonas específicas de la superficie del cuerpo de los murciélagos. Por ejemplo, las especies de la subfamilia Streblinae tienen preferencia por superficies del cuerpo cubiertas de pelo, mientras que las especies que pertenecen a la subfamilia Trichobiinae tienden a ubicarse con mayor frecuencia en las membranas de las alas al igual que las especies de ácaros de la familia Spinturnicidae (Ter Hofstede y Fenton 2005).

La especificidad filogenética, representada mediante el valor de AF, no mostró cambios significativos entre los distintos paisajes. Sin embargo, se considera que podría existir un efecto sobre la especificidad filogenética de los ectoparásitos por efectos ambientales (Dick y Dittmar 2014). Otros estudios mencionan que las especies hospederas preferidas por las moscas parásitas no corresponden con las especies consideradas adecuadas, entendiéndose a éstas últimas como las especies de hospedero en las que el parásito tiene el mayor éxito parasitario (Wenzel et al. 1966). Con tiempo y oportunidades de adaptación suficientes, el hospedero más adecuado podría convertirse en algún momento en el hospedero preferido en un área delimitada, lo que destaca que estos procesos se encuentran en constante dinamismo, con variantes espaciales y temporales.

Conocer los efectos de la transformación ambiental en las comunidades de sus ectoparásitos es primordial para el análisis y comprensión del impacto que generan las actividades antropogénicas en los agroecosistemas. En consecuencia, la consideración de factores ambientales que afecten la riqueza, abundancia y diversidad de los ectoparásitos, así como la especificidad filogenética con sus hospederos, es esencial para profundizar en el estudio de la relación hospedero-parásito. Este trabajo es pionero en la incorporación de características de paisajes fragmentados por la actividad pecuaria en el análisis de la composición de comunidades de ectoparásitos y su relación filogenética con especies de vertebrados de importancia ecológica como los murciélagos. De manera similar a lo planteado por Froeschke et al. (2013), por lo que las inferencias precisas sobre el efecto de las transformaciones del hábitat en las abundancias de los parásitos sólo serán posibles cuando las historias de vida del hospedero y parásito se estudien e involucren a fondo en el análisis.

\section{CONCLUSIONES}

La composición y configuración del paisaje pueden proporcionar información importante sobre las relaciones entre murciélagos y ectoparásitos en sitios perturbados por la actividad pecuaria, un ecosistema que anteriormente se había estudiado con poca frecuencia. El nivel de transformación de los paisajes agropecuarios definido por una variable categórica tiene un efecto sobre la composición de las comunidades de ectoparásitos asociadas con $A$. jamaicensis. El acahual, es el paisaje con menor transformación antropogénica, presentó los valores más altos de diversidad de especies de murciélagos y ectoparásitos. Los patrones de especificidad de hospedero parecen responder en mayor medida a la asociación parásito-hospedero. Por lo tanto, la composición de las comunidades de ectoparásitos depende en mayor medida de las especies hospederas presentes en la región de estudio. Se necesitan estudios que profundicen en la caracterización cuantitativa de paisajes antropizados debido a la actividad pecuaria, a fin de no depender de un sitio conservado de referencia, para facilitar las comparaciones entre sitios con paisajes transformados por actividades antropogénicas. 


\section{AGRADECIMIENTOS}

Al apoyo brindado por los proyectos DGAPAPAPIIT IV200715 y DGAPA-PAPIME PE209417. A D. Ortiz, A. Chimal, B. Maya y S. Rodríguez por su ayuda en el trabajo de campo. A M. Alonso y J. Jarillo
(Centro de Enseñanza, Investigación y Extensión en Ganadería Tropical, FMVZ-UNAM), a C. Gómez (Centro de Bachillerato Tecnológico Agropecuario No. 17) y a E. Zanatta de La Nueva Victoria, San Andrés Tuxtla por el apoyo en la logística del trabajo de campo.

\section{LITERATURA CITADA}

Abella-Medrano CA, Ibáñez-Bernal S, Macgregor-Fors I, Santiago-Alarcon D (2015) Spatiotemporal variation of mosquito diversity (Diptera: Culicidae) at places with different land-use types within a neotropical montane cloud forest matrix. Parasites and Vectors 8: 487. Doi: 10.1186/s13071-015-1086-9.

Bezerra RHS, de Vasconcelos PF, Bocchiglieri A (2016) Ectoparasites of bats (Mammalia: Chiroptera) in Atlantic forest fragments in north-eastern Brazil. Parasitology Research 115: 3759-3765.

Bininda-Emonds ORP, Cardillo M, Jones KE, Macphee RDE, Beck RMD, Grenyer R, Price SA, Vos RA, Gittleman $\mathrm{JL}$, Purvis A (2007) The delayed rise of present-day mammals. Nature 446: 507?12. https://doi.org/10.1038/ nature05634

Bolívar-Cimé B, Cuxim-Koyoc A, Reyes-Novelo E, Morales-Malacara JB, Laborde J, Flores-Peredo R (2018). Habitat fragmentation and the prevalence of parasites (Diptera, Streblidae) on three Phyllostomid bat species. Biotropica 50: 90-97

Ceballos G, Arroyo-Cabrales J (2012) Lista actualizada de los mamíferos de México. Revista Mexicana de Mastozoología. http://www.ecologia-unam.com.mx/wp-content/uploads/2016/03/Ceballos-y-Arroyo-2012.pdf. Fecha de consulta: 29 de junio de 2018.

Colín-Martínez H, García-Estrada C (2018) Parasite load and new soft tick record (Ixodida: Argasidae) on the bat species Balantiopteryx plicata and Pteronotus parnellii in Oaxaca, Mexico. Journal of Vector Ecology 43: $190-192$.

Colín-Martínez H, Morales-Malacara JB, García-Estrada C (2018) Epizoic fauna survey on phyllostomid bats (Chiroptera: Phyllostomidae) in a shaded coffee plantation of southeastern Chiapas, Mexico. Journal of Medical Entomology 55: 172-182.

Cristóbal CV, Méndez JLA, Canche JMP, Hernández ER (2014). Indicadores de diversidad, estructura y riqueza para la conservación de la biodiversidad vegetal en los paisajes rurales. Tropical and Subtropical Agroecosystems 17: 185-196.

Cuxim-Koyoc A, Reyes-Novelo E, MacSwiney MC, Pech-Canché JM (2018) Moscas ectoparásitas de murciélagos (Diptera: Streblidae y Nycteribiidae) del valle de Uxpanapa, Veracruz, México. Revista Mexicana de Biodiversidad 89: 1074-1088.

Czenze ZJ, Broders HG (2011) Ectoparasite community structure of two bats (Myotis lucifugus and M. septentrionalis) from the maritimes of Canada. Journal of Parasitology Research 2011: 1-9. doi: 10.1155/2011/3415 35.

Daily GC, Ceballos G, Pacheco J, Suzán G, Sanchez-Azofeifa A (2014) Countryside biogeography of neotropical mammals: Conservation opportunities in agricultural landscape of Costa Rica. Conservation Biology 17: 1814-1826. 
de Almeida JC, Gomes LAC, Owen RD (2017) Morphometric variation in Periglischrus torrealbai (Acari: Spinturnicidae) on three species of host bats (Chiroptera: Phyllostomidae) with a new record of host species. Parasitology Research 117: 257-264.

Dick CW, Dittmar K (2014) Parasitic bat flies (Diptera: Streblidae and Nycteribiidae): Host specificity and potential as vectors. In: Klimpel S, Mehlhorn $\mathrm{H}$ (eds) Bats (Chiroptera) as vectors of diseases and parasites. Vol. 5. Berlin: Springer-Verlag Berlin Heidelberg. pp: 131-156.

Dittmar K, Morse SF, Dick CW, Patterson BD (2015) Bat fly evolution form the Eocene to the present (Hippoboscoidea: Streblidae and Nycteribiidae. In: Morand S, Krasnov BR, Littlewood DTJ (eds). Parasite diversity and diversification: Evolutionary ecology meets phyllogenetics, Cambridge University Press, Cambridge, United Kingdom. pp. 246-264.

Dittmar K, Porter ML, Murray S, Whiting MF (2006) Molecular phylogenetic analysis of nycteribiid and streblid bat flies (Diptera: Brachycera, Calyptratae): Implications for host associations and phylogeographic origins. Molecular Phylogenetics and Evolution 38: 155-170.

Estrada-Contreras I, Equihua M, Castillo-Campos G, Rojas-Soto O (2015) Climate change and effects on vegetation in Veracruz, México: an approach using ecological niche modelling. Acta Botanica Mexicana 93: 73-93.

Frank HK, Mendenhall CD, Judson SD, Daily GC, Hadly EA (2016) Anthropogenic impacts on Costa Rican bat parasitism are sex specific. Ecology and Evolution 6: 4898-4909.

Froeschke G, van der Mescht L, McGeoch M, Matthee S (2013) Life history strategy influences parasite responses to habitat fragmentation. International Journal for Parasitology 43: 1109-1118.

Gay N, Olival KJ, Bumrungsri S, Siriaroonrat B, Bourgarel M, Morand S (2014) Parasite and viral species richness of Southeast Asian bats: Fragmentation of area distribution matters. International Journal for Parasitology: Parasites and Wildlife 3: 161-170.

Gonçalves F, Fischer E, Dirzo R (2017) Forest conversion to cattle ranching differentially affects taxonomic and functional groups of Neotropical bats. Biological Conservation 210: 343-348.

González-Acuña D, Saracho-Bottero MN, Ossa G, Guglielmone A, Nava S (2018) Ixodes chilensis Kohks, 1956 (Acari: Ixodida: Ixodidae): Re-description of the female, description of the nymph, and phylogenetic position inferred from mitochondrial DNA sequences of the 16S rRNA gene. Systematic Parasitology 9: 959-967.

Hernández-Martínez J, Morales-Malacara JB, Alvarez-Añorve MY, Amador-Hernández S, Oyama K, Avila Cabadilla LD (2018). Drivers potentially influencing host-bat fly interactions in anthropogenic neotropical landscapes at different spatial scales. Parasitology 146: 74-88.

Kazak C, Döker I, Karut K (2017) First record of invasive tomato spider mite Tetranychus evansi (Acari: Tetranychidae) in Turkey. International Journal of Acarology 43: 325-328.

Krasnov BR, Shenbrot GI, Khokhlova IS, Degen AA (2004) Relationship between host diversity and parasite diversity: Flea assemblages on small mammals. Journal of Biogeography 31: 1857-1866.

Kunz TH, de Torrez EB, Bauer D, Lobova T, Fleming TH (2011) Ecosystem services provided by bats. Annals of the New York Academy of Sciences 1223: 1-38.

López-Pérez AM, Gage K, Rubio AV, Montenieri J, Orozco L, Suzán G (2018) Drivers of flea (Siphonaptera) community structure in sympatric wild carnivores in northwestern Mexico. Journal of Vector Ecology 43: 15-25. 
Lourenço EC, Almeida JC, Famadas KM (2016) Richness of ectoparasitic flies (Diptera: Streblidae) of bats (Chiroptera) a systematic review and meta-analysis of studies in Brazil. Parasitology Research 115: 43794388.

Mackenzie K, Hemmingsen W (2014) Parasites as biological tags in marine fisheries research: European Atlantic waters. Parasitology 142: 54-67.

Medellín RA, Arita HT, Sánchez HO (2008) Identificación de los murciélagos de México, clave de campo. $2^{\mathrm{a}}$ ed. Instituto de Ecología, Universidad Nacional Autónoma de México. México. 28-51.

Medellín RA, Equihua M, Amín MA (2000). Bat diversity and abundance as indicators of disturbance in neotropical rainforest. Conservation Biology 14: 1666-1675. https://doi.org/10.1111/j.1523-1739.2000.99068.x.

Newbold T, Hudson LN, Hill SLL, Contu S, Lysenko I, Senior RA, Börger L, Bennett DJ, Choimes A, Collen B, Day J, De Palma A, Díaz S, Echeverria-Londoño S, Edgar MJ, Feldman A, Garon M, Harrison MLK, Alhusseini T, Ingram DJ, Itescu Y, Kattge J, Kemp V, Kirkpatrick L, Kleyer M, Correia DLP, Martin CD, Meiri S, Novosolov M, Pan Y, Phillips HRP, Purves DW, Robinson A, Simpson J, Tuck SL, Weiher E, White HJ, Ewers RM, Mace GM, Scharlemann JPW, Purvis A (2015) Global effects of land use on local terrestrial biodiversity. Nature 520: 45-50.

Patterson BD, Dick CW, Dittmar K (2007) Roosting habits of bats affect their parasitism by bat flies (Diptera: Streblidae). Source Journal of Tropical Ecology 23: 177-189.

Patterson BD, Dick CW, Dittmar K (2008) Parasitism by bat flies (Diptera: Streblidae) on neotropical bats: Effects of host body size, distribution, and abundance. Parasitology Research 103: 1091-1100.

Pérez-Nicolás M, Vibrans H, Romero-Manzanares A (2018) Can the use of medicinal plants motivate forest conservation in the humid mountains of Northern Oaxaca, Mexico?. Botanical Sciences 96: 267-285.

Pilosof S, Dick CW, Korine C, Patterson BD, Krasnov BR (2012) Effects of Anthropogenic disturbance and climate on patterns of bat fly parasitism. Plos One 7: 1-7 e41487. Doi: 10.1371/journal.pone.0041487.

Poulin R, Krasnov BR, Mouillot D (2011) Host specificity in phylogenetic and geographic space. Trends in Parasitology 27: 355-361

Presley SJ (2010) Interspecific aggregation of ectoparasites on bats: importance of hosts as habitats supersedes interspecific interactions. Oikos 120: 832-841.

Ramírez-Pulido J, González-Ruiz N, Gardner AL, Arroyo-Cabrales J (2014) List of recent land mammals of Mexico, 2014. Special Publications Museum of Texas Tech University 63: 1-69.

Rico-Chávez O, Ojeda Flores R, Sotomayor Bonilla J, Zambrana-Torrelio C, Loza Rubio E, Alonso Aguirre A, Suzán $\mathrm{G}$ (2015) Viral diversity of bat communities in human-dominated landscapes in Mexico. Veterinaria México 2: 1-22.

Ries L, Fletcher RJ, Battin J, Sisk TD (2004) Ecological responses to habitat edges: mechanisms, models, and variability explained. Annual Review of Ecology Evolution and Systematics 35: 491-522

Russo D, Jones G (2015) Bats as indicators: an introduction. Mammalian Biology 80: 157-158.

Sikes RS, Bryan JA (2016) Guidelines of the American Society of Mammalogists for the use of wild mammals in research and education. Journal of Mammalogy 97: 663-688.

Suzán G, Marcé E, Giermakowski JT, Armién B, Pascale J, Mills J, Ceballos G, Gómez A, Aguirre AA, SalazarBravo J, Armién A, Parmenter R, Yates T (2008) The effect of habitat fragmentation and species diversity loss on hantavirus prevalence in Panama. Annals of the New York Academy of Sciences 1149: 80-83. 
Tello JS, Stevens RD, Dick CW (2008) Patterns of species co-occurrence and density compensation: A test for interspecific competition in bat ectoparasite infracommunities. Oikos 117: 693-702.

Ter Hofstede HM, Fenton MB (2005) Relationships between roost preferences, ectoparasite density, and grooming behaviour of neotropical bats. Journal of Zoology 266: 333-340.

Tlapaya-Romero L, Horváth A, Gallina-Tessaro S, Naranjo E, Gómez B (2015). Prevalencia y abundancia de moscas parásitas asociadas a una comunidad de murciélagos cavernícolas en La Trinitaria, Chiapas, México. Revista Mexicana de Biodiversidad 86: 377-385.

Trilleras JM, Jaramillo VJ, Vega EV, Balvanera P (2015) Effects of livestock management on the supply of ecosystem services in pastures in a tropical dry region of western Mexico. Agriculture, Ecosystems and Environment 211: 133-144.

Voigt CC, Kingston T (2016) Bats in the Anthropocene. En: Voigt CC, Kingston T (eds) Bats in the anthropocene: Conservation of bats in a changing world. New York: Springer International Publishing. pp: 1-9

Wenzel RL (1976) The Streblid batflies of Venezuela (Diptera: Streblidae). Brigham Young University Science Bulletin Biological Series 20: 1-177.

Wenzel RL, Tipton VJ, Kiewlicz A (1966) The streblid batflies of Panama (Diptera: Streblidae). In: Wenzel RL, Tipton VJ (eds). Ectoparasites of Panama, Field Museum of Natural History, Chicago, pp: 405-675. 